\title{
"Vivir en dictadura". La historia oral y la enseñanza del pasado reciente ${ }^{1}$ Benadiba, Laura \\ Plotinsky, Daniel \\ Universidad de Buenos Aires
}

\section{"Memoria e historia, \\ ¿No están condenadas a la cohabitación forzosa?"2}

Si partimos del acuerdo tácito de que en nuestro país "historia reciente" refiere a la militancia político-social y las movilizaciones de masas desarrollada durante las décadas del ` 60 y 70 y al terrorismo de Estado instaurado por la dictadura militar, ${ }^{3}$ para quienes trabajamos en la educación media desde hace dos décadas las dificultades que genera su introducción en las aulas evoca diversas etapas, situaciones, errores y estados de ánimo.

Resulta inevitable comenzar este trabajo recordando la angustia que nos generaba el absoluto silencio de los alumnos cuando a fines de los años ` 80 tratábamos de introducir el tema de los "hijos apropiados" durante la dictadura, y el tiempo transcurrido hasta descubrir que ese silencio podía no ser una señal de desinterés adolescente sino un síntoma del temor que les provocaba la posibilidad de ser uno de esos chicos apropiados.

El paso del tiempo confirmó nuestra apreciación, siendo hoy uno de los temas que genera mayor interés entre los alumnos.

En este sentido, el presente trabajo parte del entendimiento de que los "problemas" y "dificultades" generados por la inclusión de la "historia tiempo presente" en la escuela son diferentes según se piensen desde el ámbito de los historiadores o desde el ámbito escolar.

En principio, está el amplio debate acerca de las relaciones entre historia y memoria y en torno a los conceptos historia reciente, historia tiempo presente, historia coetánea, historia del pasado inmediato, memoria histórica. ${ }^{4}$

Sin entrar en el mismo, consideramos que la dictadura militar que se apropió del poder en nuestro país entre 1976 y 1983 coincide con los criterios de periodización de la historia presente que "han partido habitualmente de acontecimientos nodales, de hechos fundadores ligados a una gran convulsión política (una guerra, una revolución...) que afecta de manera decisiva a una determinada historia nacional".

Por otra parte, y de alguna manera vinculado con aquellos debates, está el análisis sobre las características de la producción bibliográfica sobre nuestro pasado reciente y las posibilidades de su utilización en la escuela. ${ }^{6}$ 
Pero aún al interior de la escuela, cohabitan distintos problemas y dificultades.

Por una parte, la existencia de un "currículo visible" -expresado, entre otros elementos, en políticas educativas, planificaciones y libros de texto- y uno oculto, condicionado por la experiencia y prácticas culturales de los miembros de la comunidad educativa: alumnos, docentes, familias.

Por otra, las propias divergencias entre las experiencias de vida de alumnos, docentes y padres, que se observan diariamente en la escuela pero que resulta "especialmente notorio frente a temas teñidos de un fuerte tono ético moral, o que no admiten posturas neutras o distancias críticas por la proximidad histórica o el involucramiento personal".?

Nos centraremos, entonces, sólo en algunos aspectos de la vasta problemática que el tema motiva: cuales son los aportes de la historia oral a la enseñanza de la historia reciente a partir del examen de una experiencia desarrollada desde 2006 en la Escuela Técnica ORT ${ }^{8}$ en el marco de las actividades del Proyecto Archivo Oral: el Taller "Vivir en Dictadura".

\section{"Vivir en dictadura"}

El taller extracurricular "Vivir en Dictadura", que surgió como parte de las actividades planificadas en la Escuela para recordar los 30 años del Golpe de Estado, propone a los alumnos trabajar a partir de tres ejes:

- El análisis y la reflexión acerca de la vida cotidiana de los argentinos durante la última dictadura militar (1976/1983).

- El diseño y desarrollo de un proyecto de investigación basado fundamentalmente en la utilización de técnicas cualitativas.

- La creación y utilización de fuentes orales en un contexto de investigación.

La idea de llevar a cabo este Taller se fundamentó en la significatividad y relevancia del tema propuesto, la pertinencia de la metodología a desarrollar y nuestra experiencia previa en el trabajo de creación y utilización de fuentes orales por parte de los alumnos.

a) El tema:

Desde el retorno a la legalidad democrática en 1983 el análisis del período dictatorial ha sido encarado desde múltiples ópticas y con diversas intenciones. Sin embargo, quedan anchas franjas de la realidad que esperan ser analizadas, tal como lo demuestran las polémicas abiertas alrededor de la recordación del 30vo. aniversario del Golpe de Estado.

El taller propone abordar una de las áreas de la realidad social menos transitadas en la escuela: ¿Cómo era la vida cotidiana durante la dictadura? ¿Qué pasaba en las casas, las escuelas, las oficinas, la calle? ¿Cómo se reflejaba en las relaciones familiares?

Porque "la gente iba a trabajar, viajaba en colectivo, en taxi, en tren, cruzaba calles, caminaba por las veredas. El sol salía y había luz y hasta algunos días de otoño eran cálidos. ¿Dónde estaba el horror? Había señales: los policías usaban casco, en los aeropuertos había muchos soldados, sonaban sirenas. Los militares les hacían sentir a los ciudadanos que estaban constantemente 
en operaciones en medio de una "guerra". Pero, a la luz del día, nada parecía tan espantoso como sabíamos que era. Quiero remarcar esta sutil y terrible vivencia del horror: lo cotidiano como normalidad que oculta la latencia de la Muerte".

Partimos de definir lo "cotidiano" como el ámbito concreto en el que se define el modo de vida de los sujetos, la sedimentación de un conjunto de actividades y actitudes, rutinas y hábitos que se mantienen durante un período prolongado de tiempo. ${ }^{10}$ Poder internarse -a través de las entrevistas- en ese "cotidiano" permite entonces preguntarse "cómo fue que la experiencia cotidiana del horror y el terror se volvió, valga la redundancia, cotidiana; qué prácticas, sentimientos e imaginarios la constituyeron y la sostuvieron; cuándo comenzó y cuándo se interrumpió la continuidad; y qué hay de continuidad, de discontinuidad y de ruptura, desde la memoria de los sujetos, entre el pasado y el presente". ${ }^{11}$

b) La propuesta metodológica:

Fomentar la investigación y el pensamiento crítico y autónomo de los alumnos es uno de los objetivos de la escuela media. Por eso, el taller se propone brindar un ámbito en el que los participantes puedan experimentar los métodos, técnicas y habilidades necesarias para desarrollar y culminar exitosamente un proyecto de investigación.

Los proyectos deben basarse en el trabajo con fuentes orales ya que consideramos que el mismo, tanto en su etapa de creación como en la de su utilización, ayuda a los alumnos a comprender las características del conocimiento histórico y del trabajo del investigador.

El proceso de preparación de las entrevistas, que requiere capacitar a los alumnos en las técnicas de la entrevista de historia oral, aporta al aprendizaje utilización de otras fuentes. Esto es así porque la entrevista - por su propia esencia interactiva- permite desnudar que todo conocimiento es respuesta a una pregunta, lo que promueve un papel activo del alumno-investigador frente a todo documento y le ayuda a comprender que para conocer el pasado es necesario interrogarlo.

La experiencia misma de las entrevistas acerca a los adolescentes al trabajo concreto de investigación y les permite de un modo efectivo comprender la naturaleza de las fuentes históricas de cualquier tipo: estas no hablan por sí solas sino que, por el contrario, es imprescindible partir de hipótesis y formularles preguntas.

Por otra parte, las entrevistas facilitan la generación de condiciones para que los alumnos puedan acceder a las experiencias de la vida cotidiana ya que crean las bases para un proceso de identificación y una ampliación inter-generacional del "nosotros", requisito indispensable para poder transmitir las interpretaciones y explicaciones del pasado de una generación a otra. En este sentido, el Proyecto permite unir dos subgrupos sociales:

1. El de los informantes que "vivieron" la dictadura. "Para ellos, esa vivencia puede ser un hito central de su vida. Si se trató de un acontecimiento traumático, puede ser un hueco, un vacío, un silencio, o las huellas de ese trauma manifiestas en conductas actuales (pocas veces, sin embargo, un simple "olvido")": ${ }^{12}$

2. El de los alumnos, "que no tienen esa "la experiencia pasada" propia. Esta falta de experiencia los pone en una aparente otra categoría: son los otro /as, los que no lo vivieron en carne 
propia. Para este grupo, la memoria es una visión del pasado construida como conocimiento cultural compartido por generaciones sucesivas y por diversos otro/ as." ${ }^{13}$

Finalmente, la utilización de las fuentes orales en la elaboración de los trabajos, confrontando diferentes testimonios entre sí y con la información que surge de otro tipo de fuentes, ayuda a transparentar alguna de las características de los conceptos sociales e históricos: ser relativos, cambiantes en el tiempo, sufrir la influencia cultural e ideológica del medio y, finalmente, ser subjetivos en tanto producto humano.

Se genera así otra de las condiciones necesarias para que la transmisión de las experiencias sea exitosa: dejar abierta la posibilidad de que quienes las reciben le den su propio sentido, las reinterpreten y resignifiquen, y no que repitan o memoricen.

c) El Archivo Oral:

El desarrollo de este taller se enmarca en el trabajo realizado por el Archivo Oral de la Escuela y se articula con el resto del trabajo realizado en el marco del mismo.

El Proyecto Archivo Oral de la Escuela Técnica ORT lleva a cabo desde 1993 experiencias de trabajo para la creación de Archivos Orales en los que se propone preservar de manera organizada las experiencias sociales de las familias de ORT a través de entrevistas video-grabadas, realizadas por un grupo de alumnos voluntarios dirigido por un equipo docente.

Los objetivos generales del Proyecto son:

1. Despertar la curiosidad de los alumnos, logrando que valoricen el reconocimiento de problemas y la búsqueda de respuestas propias.

2. Enseñar una historia con protagonistas, eludiendo al mismo tiempo:

- la visión tradicional, que reduce el tema al estudio de la actuación de los grandes personajes.

- una visión despersonalizada de la historia, en la que los actores sociales son meras abstracciones alejadas de la vida -y por lo tanto de las posibilidades de comprensión- de los alumnos.

3. Elaborar estrategias que permitan superar las dificultades que los alumnos tienen para la comprensión de los conceptos históricos y sociales, considerando que gran parte de esas dificultades surgen de las características propias de esos conceptos. ${ }^{14}$

4. Lograr una propuesta de trabajo escolar que recoja la complejidad y riqueza de la realidad social -en el espacio y en el tiempo- y a la vez la presente de manera atractiva para los alumnos.

5. Conciliar propuestas curriculares que pongan el acento en el ámbito local con la necesidad de alcanzar la comprensión de los procesos sociales más generales, a escala nacional y mundial.

6. Generar conciencia a través de apreciar la utilidad de los archivos, en el camino de modificar pautas de conducta de una sociedad que -como la argentina- no valoriza la conservación de las huellas de su pasado en ninguna de sus expresiones.

El Archivo Oral de la Escuela Técnica ORT está compuesto por las filmaciones de las entrevistas que realizan los alumnos. Esto es así porque es la mejor forma de poder mantener en el tiempo toda la riqueza que tiene este dialogo entre generaciones: podemos escuchar lo dicho, pero también el tono en el que fue dicho, las emociones que produjo en el entrevistador $\mathrm{y}$ en el entrevistado, los gestos y actitudes, etc. ${ }^{15}$ 
El primer proyecto, comenzado en 1993 y aun en curso, fue el "Archivo oral, fotográfico y epistolar de las experiencias migratorias de las familias de ORT” en el que ya están preservadas más de 400 entrevistas.

La elección de esta temática obedeció a la enorme significación que el fenómeno de la inmigración representa para el conjunto de la sociedad argentina y a la importancia que la experiencia migratoria ha tenido para los protagonistas y sus descendientes.

Durante el año 2000, coincidiendo con las celebraciones por los 120 años de ORT, comenzó a desarrollarse un nuevo proyecto de trabajo para la creación de un "Archivo Histórico de ORT Argentina" que se propone documentar la historia de las Escuelas ORT, sus docentes y alumnos y el contexto institucional y comunitario en que las mismas se insertaron. Este Archivo cuenta hoy con 80 entrevistas.

Un Archivo con estas características resulta interesante para nuestros alumnos y valioso para ellos, los docentes y sus familias ya que:

a) Las fuentes que lo conforman son

- Significativas para los alumnos, ya que refieren a temas que les son familiares y a un ámbito en el que pasan gran parte de su tiempo.

- Relevantes para los contenidos curriculares, pues si bien aluden fundamentalmente a la historia de la escuela, remiten al conocimiento de sucesos y procesos sociales que la acompañan y explican. ${ }^{16}$

b) Reconocer como fuente histórica la experiencia escolar de las generaciones pasadas contribuye a sentirse parte integrante de la comunidad educativa y a crear responsabilidad sobre los actos del presente en tanto todos los que participan del proyecto se transforman en posibles referentes de las generaciones futuras.

c) Rescatar el pasado de la institución escolar como memoria social colabora en la conformación de una "identidad colectiva", común a todos los integrantes de la comunidad educativa. Trabajar con la memoria institucional recuperada hace posible inventariar el pasado colectiva e individualmente, reconocerse con historia y valorar el presente. En ese sentido, opera como una forma de encontrar valores, propuestas, proyectos inconclusos y sentidos de pertenencia, al mismo tiempo que ayuda a contextualizar y desidealizar recuerdos y tradiciones.

Finalmente, en el marco de las actividades desarrolladas en 2006 para rememorar los 30 años del Golpe de Estado comenzó el trabajo del proyecto "Vivir en dictadura", del que participan en este momento 35 alumnos.

\section{La experiencia desarrollada}

En tanto nos encontramos con un proyecto en plena ejecución, la evaluación de la actividad realizada durante estos dos años es provisoria. Sin embargo, podemos afirmar que los objetivos iniciales se están cumpliendo con resultados que -en algunos aspectos- superan nuestras expectativas más optimistas.

En primer lugar, si compartimos con Brunello ${ }^{17}$ que una de las dificultades para generar el 
interés escolar por la historia radica en que los estudiantes no tienen confianza en el futuro, y que la demanda de la historia nace cuando existe un proyecto compartido y se advierten algunos vínculos entre las dimensiones temporales pasado-presente-futuro, podemos afirmar que el taller ha generado un particular clima de trabajo que favorece el proceso de involucramiento colectivo.

Esto es así, entre otras cosas, porque los proyectos basados en la creación y utilización de fuentes orales tienen un enorme potencial para cambiar la relación de la gente con su pasado y con el futuro. "Pueden sacar el pasado del dominio de lo trivial y nostálgico y comenzar a generar la conciencia de la historia como el relato de la acción humana, las elecciones humanas". ${ }^{18}$

Al preparar, realizar y analizar las entrevistas, los alumnos recorren el camino que lleva a la experiencia individual partiendo de su contexto social y familiar, atravesando una red de mediaciones que a su vez les sirven para invertir el movimiento y alcanzar el universo histórico y social a partir de un individuo. La contextualización es la trama en la que se insertan y se encuadran las diferentes historias de vida, adquiriendo todo el significado de indispensables fragmentos del mosaico social general. ${ }^{19}$

Por otra parte, la construcción y utilización de fuentes orales tiene un indudable interés en la búsqueda de raíces y señas de identidad colectivas, por lo que el proyecto contribuye a identificar las huellas del pasado y a integrar al alumno en su propio medio a través del reconocimiento crítico del mismo, en la larga y la corta duración.

En este sentido, los testimonios sobre la vida cotidiana durante la dictadura y la exploración sobre las condiciones que permitieron que esta se establezca generan las condiciones para reflexionar sobre las características de la cultura político-social previa a ella, evitando tanto la condena anacrónica de esa cultura como su reivindicación nostálgica. ${ }^{20}$

Las investigaciones basadas en los testimonios recogidos, además, hacen más factible aplicar un enfoque histórico al estudio de la realidad cercana. "Más que ayudar a la construcción de una historia "reciente", "inmediata", o "del tiempo presente", que no puede tener entidad sin dar cabida a "la explicación genético-estructural de unas formas sociales que están vigentes", contribuye a que sea viable una historia realmente contemporánea, que se haga cargo efectivamente de una interacción entre pasado y presente" ${ }^{21}$

En ese contexto, las relaciones que se establecen entre los entrevistados, portadores de la experiencia vivida, y los alumnos que reciben de una manera activa el recuerdo elaborado desde el presente de esa experiencia, les permite a estos apropiarse reflexivamente del pasado expresado en los testimonios. Sólo así, ese pasado representa realmente para los adolescentes, una "historia reciente".

A los docentes, por otra parte, el proyecto nos permite involucrarnos abiertamente en el tema en nuestro doble carácter de responsables del trabajo que realizan los alumnos y sujetos de estudio -en tanto hemos "vivido en dictadura" - lo que nos permite compartir con ellos nuestras propias tensiones, contradicciones y formas de resignificar el pasado.

Desde ese lugar, podemos evitar dilemas habituales en el aula: ni "bajamos linea", ni pretendemos una neutralidad valorativa que, como observa Sandra Raggio, ante su imposibilidad real termina traduciéndose en un silencio prolongado o el "recuerdo efeméride". ${ }^{22}$ 
En cuanto al desarrollo del trabajo, las fuentes orales, al cumplir funciones exploratorias, analíticas, verificativas y expresivas, ${ }^{23}$ permiten a los alumnos encarar proyectos de diferentes características y con diversos productos finales.

Los proyectos grupales se centran en diferentes actores sociales (docentes, periodistas, músicos, militantes políticos y sociales, etc.) y su producción se expresa en monografías, documentales y exposiciones.

El proyecto a generado, además, la posibilidad de un trabajo conjunto con los alumnos del Instituto de Educación Secundaria (IES) El Morell, de la localidad de Tarragona (Catalunya, España) en el marco del proyecto ARCA (Argentina- Cataluña). El tema de investigación del proyecto es "la persistencia del silencio" después de las dictaduras en España y Argentina.

En síntesis, consideramos que el trabajo realizado en el Taller "Vivir en dictadura" genera una práctica escolar que supone transitar un complejo camino entre la experiencia vivida y la experiencia de aprender, que podríamos sintetizar en el proceso: transmitir, legar, heredar, recibir, resignificar, aprender.

Sin embargo, somos concientes de que "las aportaciones que las Fuentes Orales pueden suministrar a la enseñanza- aprendizaje de la historia no son automáticas, no se desprenden de su mera introducción al aula, sino que dependen de los planteamientos teóricos y metodológicos en los que se articula su instrumentación. Lo mismo que ocurre con otros recursos didácticos". ${ }^{24}$

Esto significa que el éxito de un proyecto basado en la creación y utilización de fuentes orales en la escuela como el aquí propuesto se evalúa en términos escolares y depende, fundamentalmente, de dos factores:

a) Una sólida formación conceptual y metodológica de los docentes y alumnos acerca de la Historia Oral. Comenzar el proyecto careciendo de los conocimientos necesarios solo puede concluir en el fracaso del mismo, tanto sea por abandono del trabajo como porque se desvirtúen los objetivos inicialmente propuestos.

b) Un contexto escolar coherente con las potencialidades educativas de las fuentes orales.

Si esto no es así, el proyecto está condenado al fracaso o a sobrevivir como una planta exótica, muy bonita pero incapaz de reproducirse y dar frutos. Si el contexto escolar es afín al trabajo propuesto, no solo permitirá su desarrollo sino que podrá realimentarse y fortalecerse con el mismo. ${ }^{25}$ 


\section{Notas}

${ }^{1}$ Versión presentada en la XIo Jornadas Interescuelas - Departamentos de Historia realizada en la Universidad Nacional de Tucumán los días 19 al 22 de septiembre de 2007.

${ }^{2}$ Ricoeur (2000) p. 512.

${ }^{3}$ Oberti y Pittaluga (2004-2005) pp. 1-2.

${ }^{4}$ LeGoff (1991), Mateos (1998), Huyssen (2001), Arostegui (2004), Ricoeur (2004).

${ }^{5}$ Mateos (1998) p. 2.

${ }^{6}$ De Amézola (2000), Reta y Pescader (2002).

${ }^{7}$ Jelin y Lorenz (2004) p. 4.

${ }^{8}$ Escuela de educación media ubicada en la Ciudad Autónoma de Buenos Aires.

${ }^{9}$ Feinman (1998)

${ }^{10}$ Lechner (1988) p. 44-53.

${ }^{11}$ Caviglia (2006) p. 55.

${ }^{12}$ Jelin (2000) p. 8.

${ }^{13}$ Jelin (2000) p. 8.

${ }^{14}$ Carretero y Limón (1997) pp. 37-44.

${ }^{15}$ Voldman (1991)

${ }^{16}$ Vezub (1994)

${ }^{17}$ Brunello (1989) p.130

${ }^{18}$ Shopes (1993) p. 251

${ }^{19}$ Ferrarotti (1990) p. $9-12$

${ }^{20}$ Sábato (2001) p. 44.

${ }^{21}$ Borras Llop (1989) p. 150.

${ }^{22}$ Raggio (2002) p. 44 - 45.

${ }^{23}$ Bertaux (1989) p. 93.

${ }^{24}$ Borras Llop (1989) p.144.

${ }^{25}$ Benadiba y Plotinsky (2001) pp. 119-120. 


\section{Bibliografía}

Aceves Lozano, Jorge (1993) Historia oral, Instituto Dr. José María Luis Mora, México.

Arostegui, Julio (2004) La historia vivida: sobre la historia del tiempo presente, Alianza, Madrid.

Benadiba, Laura y Daniel Plotinsky (2001) Historia Oral. Construcción del archivo histórico escolar. Una herramienta para la enseñanza de las ciencias sociales, Novedades Educativas, Buenos Aires.

Bertaux, Daniel (1989) “Los relatos de vida en el análisis social”, en Historia y fuente oral, № 1 , Barcelona, pp. 91-101.

Borras Llop, José María (1989) "Fuentes orales y enseñanza de la historia. Aportaciones y problemas", en Historia y fuente oral, № 2, Barcelona, pp. 145-160.

Brunello, Piero, (1989) "Testimonios orales y enseñanza de la historia en la escuela elemental y media en Italia", en Historia y Fuente oral, № 2, Barcelona, pp. 129-144.

Carretero, Mario (1997) Construir y enseñar las ciencias sociales y la historia, Aique, Buenos Aires.

Carretero, Mario y Limón Margarita "Construcción del conocimiento y enseñanza de las ciencias sociales y la historia", en Carretero (1997), Construir y enseñar las ciencias sociales y la historia, Aique, Buenos Aires, pp. 33-62.

Caviglia, Mariana (2006) Dictadura, vida cotidiana y clases medias. Una sociedad fracturada, Prometeo, Buenos Aires.

De Amezola, Gonzalo (2000) "Problemas y dilemas en la enseñanza de la historia reciente", en Entrepasados. Revista de historia, № 17, Buenos Aires.

Feinman, José Pablo (1998) La sangre derramada, Seix Barral, Buenos Aires.

Ferrarotti, Franco (1990) La historia y lo cotidiano, CEAL, Buenos Aires.

Huyssen, Andreas (2001) En busca del futuro perdido. Cultura y memoria en tiempos de globalización, Fondo de Cultura Económica, Buenos Aires.

Jelin, Elizabeth y Lorenz Federico (comp.) (2004) Educación y memoria. La escuela elabora el pasado, Siglo XXI, Madrid.

Lechner, Norbert (1988) "Estudiar la vida cotidiana", en Los patios interiores de la democracia, Fondo de Cultura Económica, Santiago de Chile. 
Le Goff, Jacques (1991) El orden de la memoria, Paidós, Barcelona.

Mateos, Abdón “Historia, memoria, tiempo presente", en Hispania Nova N01, 1998- 2000.

Oberti, Alejandro y Roberto Pittaluga. "Temas para una agenda de debate en torno al pasado reciente", en Políticas de la Memoria. Anuario de información e investigación del CeDinci, № 5 , Buenos Aires, 2004/2005. pp. 9-14.

Raggio, Sandra, (2002) "Cuando la escuela da la palabra”, en Puentes N0 7, La Plata, pp. 44 - 46.

Reta, María y Carlos Pescader (2002) "Representaciones del pasado reciente. Análisis de los textos escolares del nivel medio", en Clío \& Asociados. La historia enseñada, Año 6, № 6, Santa Fe.

Ricoeur, Paul (2000) La memoria. La historia. El olvido, Fondo de Cultura Económica, Buenos Aires.

Sábato, Hilda (2001) "Las preguntas sobre lo traumático", en Puentes N0 2, La Plata, pp. 44-46.

Shopes, Linda "Mas allá de la trivialidad y la nostalgia: contribuciones a la construcción de una historia local", en Aceves Lozano (1993) Historia oral, Instituto Dr. José María Luis Mora, México, pp. 243-253. Vezub, Lea "La selección de contenidos curriculares: Ios criterios de significatividad y relevancia en el conocimiento escolar. Apuntes para la selección de contenidos de historia", en Entrepasados № 7 , Buenos Aires, 1994, pp. 151-166.

Voldman, Daniele (1991) "Historia y fuente oral en Francia a finales de los años ochenta", en Historia y Fuente Oral, № 5 , Barcelona. 